\title{
Cuban Health Professionals: Will Publishing Perish?
}

\section{By Francisco Rojas Ochoa, MD, PhD}

Cuba is a small country with one of the highest physician-percapita ratios in the world (1:155 in 2007). Since the 1980s, all new MDs are required to complete a family medicine residency, and many go on to pursue a second medical specialty or an advanced degree in public health, medical education or medical sciences. The numbers of other university-level health professionals are impressive. Cuba is also home to globally-respected biotechnology and medical research institutions, such as the Finlay Institute and the Pedro Kourí Institute of Tropical Medicine.

Yet, the number and frequency of articles by Cuban authors published in national and international journals does not reflect the breadth and depth of Cuban medical research and practice. The most frequently cited articles in international journals of impact are in the fields of genetics, neurosciences, immunology, pharmacology and oncology, and almost $80 \%$ of these are co-authored by non-Cubans. While it is certain that many of these articles reflect public health applications, you will find scant citations of articles on other important topics at the forefront of Cuban public health-such as primary health care, infectious disease control, human resource development, sexual and reproductive health, and social determinants of health and wellness.

Why is this so?

Some say Cuban doctors and other health professionals are already overworked, teaching or serving as administrators in addition to delivering health care. Some blame the lack of resources. Others claim that scientific writing has never recovered from the low priority it was given during the severe economic crisis of the 1990s, when even medications were in short supply. Still others suggest lack of writing ability - which, ironically, can only be acquired by writing or by re-writing when mandated by a journal's editors.

Ultimately, however, one answer may encompass all of the above and thus be closest to the truth: in the Cuban scientific community today, writing for publication is undervalued.

How, then, can our professionals be encouraged to publish?

First, by demonstrating that the greatest value of publishing is what the author learns in the process. Publishing makes for better health professionals. Each writing exercise obligates the author to know the subject thoroughly. In order to write, you must investigate-in the laboratory and the clinic, out in the field, in a library or on the Internet. The result will be not only what you write, but also what you learn.

Thus, the art of scientific writing should be taught early in the university-level health sciences curricula. Student science fairs were once a great opportunity for teaching: under the guidance of their professors, students presented their own research and writing. In other words, they learned by doing. Later, however, the use and abuse of posters, PowerPoint presentations and other audiovisual aids largely displaced the writing exercise itself-to the detriment of future health professionals.

The situation is better at the graduate level, since a thesis is required of residents and master's degree candidates, and the dissertation is fundamental for doctoral degrees. But publication is only required for doctoral candidates, and then just two articles. Graduate students are rarely assigned an essay or revision article on a relevant topic, even though this practice is valuable for teaching and evaluation, and also helps develop the skills needed to prepare an article for publication.

Second, by imparting the idea that scientists are best known by their published work. Their publications are valuable personal references. In this respect, editors of Cuban medical journals must share the responsibility for encouraging authors to publish by demanding quality manuscripts, working with writers, and assuring their publications are peer-reviewed. This will also lead to more Cuban medical journals accepted for index-

\section{In the Cuban scientific community today, writing for publication is undervalued}

ing by services such as SciELO, increasing international exposure for Cuban authors in the process.
Third, by extending more support to authors across the country. This includes offering more courses and workshops on scientific writing, particularly outside the capital city, where there is great potential. It also means making reference works available in print and online, and publicizing this availability, so that writers have greater access to materials, such as the Vancouver guidelines and Robert Day's How to Write and Publish a Scientific Paper, that assist them in preparing articles that meet international standards.

Departments should be established in our educational and scientific research institutions, with the express purpose of stimulating the production of scientific literature and providing logistical support for writers. Authors' institutions should take additional responsibility for disseminating the results of their work, thereby extending the writer's and the institution's contribution to the scientific community and society as a whole.

Science needs publishing. Research results; new ideas on key problems debated by the international scientific community; and public presentation of hypotheses, methods and findings are all an important part of scientific work. Without debate and discussion, we limit the advances made by research and the application of its results. Discussion is an essential element of publishing, just as publishing is essential to generating further research to confirm or rectify previous findings. In the medical sciences, publication is essential to knowledge sharing for better health and greater health equity.

Publish or perish? No. But let's not perish the need to publish. - 